\title{
Des névroses à une nouvelle cure des âmes : C. G. Jung et la refonte du patient
}

\section{thérapeutique.}

\section{Sonu Shamdasani* Londres}

En 1922, l'ethnologue et linguiste Jaime de Angulo lançait « un défi à tous ses "frères en névrose" - Allez mes frères, allez à la Mecque, je veux dire à Zürich, et buvez à la fontaine de vie, vous tous qui êtes morts en vos âmes, allez et cherchez une vie nouvelle ${ }^{1} »$. C'est évidemment vers Jung que De Angulo exhortait ses frères en névrose à se tourner. En 1912, la célébrité de Jung était telle qu'un nombre croissant de névrosés faisaient route jusqu'à lui. De Angulo et ses frères en névrose ne recherchaient point dans la psychothérapie de Jung une guérison d'ordre médical, mais le moyen de s'extraire d'un malaise culturel et spirituel largement répandu.

Au cours des dernières décennies du dix-neuvième siècle, alignée sur la pratique médicale générale, la psychothérapie à ses débuts et sous ses diverses formes, avait cautionné une conception privative de la santé comme étant l'absence de désordres nerveux et psychologiques. Ceci entraîna une vision négative du but de la psychothérapie, qui consistait à se défaire de la pathologie et à retrouver une vie normale. Les écoles de psychothérapie véhiculaient certes des conceptions diverses des troubles mentaux ainsi que des moyens d'en venir à bout, mais elles avaient généralement en commun une telle vision. A partir de la première guerre mondiale, Jung commença à s'en distancer et à reformuler la pratique de la psychothérapie comme visant dans la perspective de

\footnotetext{
*Sonu Shamdasani est

${ }^{1}$ Jaime de Angulo à Chauncey Goodrich, 28 août 1922, Goodrich papers, Bancroft library, University of California at San Francisco.
} 
l'accroissement du développement spirituel de l'individu.

Cette reformulation allait avoir de vastes retentissements sur les développements ultérieurs de la psychothérapie, tout autant que sur la pléthore de thérapies humanistes, new age et alternatives qui voyaient le jour. Tout en affirmant que sa psychothérapie se rangeait du côté de la psychologie «médicale », Jung proposait une définition nouvelle de l'humain, qui emporta l'adhésion de nombreux individus. Il n'était pas le seul à proposer une dimension méliorative. À partir des années trente, nombre d'auteurs dans ce champ en firent autant. De façon significative, ces reformulations étaient accompagnées de conceptions qui se faisaient concurrence quant à savoir ce qu'être humain voulait dire. Les pages qui vont suivre sont donc à envisager comme une étude de cas au sein d'une transformation globale du champ ${ }^{2}$.

En se démarquant d'une vision médicale conventionnelle des buts de la psychothérapie, Jung ouvrit la voie à la question des relations entre psychothérapie et pratiques religieuses. En 1904, Pierre Janet avait observé que lorsque les patients trouvaient un ami ou quelqu'un à qui obéir, leurs problèmes cessaient. Les prêtres avaient rempli cette fonction par le passé et les médecins pouvaient le faire à présent. Les prêtres l'avaient fait de façon spontanée ; cependant, ils ne détenaient plus l'autorité qui avait été la leur. Et Janet de noter que «c'est une caractéristique de notre temps que ce travail de direction morale soit parfois effectué par un médecin à qui est souvent attribué ce rôle de direction morale lorsque le patient ne trouve plus suffisamment de soutien autour de lui ${ }^{3}$.

En 1912, Jung étudie les parallèles entre la psychanalyse et la pratique de la confession. Il affirme que la valeur psychologique de la confession réside dans le fait qu'elle permet à l'être en souffrance de sortir de son isolement pour réintégrer la communauté humaine et pour instituer un lien moral qu'il identifie au « transfert » psychanalytique. La valeur morale que l'Église attribue à la confession est justifiée par le fait que « la plus grande partie de l'humanité n'avait pas seulement

\footnotetext{
2 Pour l'étude de ce contexte, voir "Psychotherapy in society: historical reflections", The Routledge Encylopedia of Madness and Mental Health, édité par Greg Eghighan, New York, Routledge, 2017, p. 363-378.

${ }^{3}$ Pierre Janet, Les obsessions et la psychasthénie, Paris, Alcan, I, 1903, p. 727.
} 
besoin de conseils, mais souhaitait surtout être guidée et mise sous tutelle ${ }^{4} »$. À travers la confession, le prêtre se substituait aux parents et aidait l'individu à se libérer de sa famille. Il en alla ainsi durant mille cinq cents ans, au cours desquels la confession s'avéra un outil éducatif efficace. Cependant, pour l'individu contemporain, elle perdit de son efficacité éducative lorsque l'église « dès que l'église s'avéra incapable de maintenir son leadership dans la sphère intellectuelle ${ }^{5}$ ».

L'individu moderne voulait comprendre et refusait de sacrifier l'intellect, son but étant d'atteindre l'autonomie morale et d'être capable de mener sa vie.

Confronté à cette exigence, le médecin se devait d'analyser le transfert, ce que le prêtre n'avait pas eu à faire. Ainsi, selon Jung, le déclin de la confession créa un contexte propice à la possibilité de la psychanalyse, qui se présentait comme une cure moderne de l'âme.

Si le médecin se devait d'occuper une place de plus en plus désertée par les prêtres, ceci posait de nouveau la question du rôle de la suggestion en psychothérapie. Durant la première décennie du vingtième siècle, une protestation s'était élevée contre l'usage de la suggestion et de l'hypnose en psychothérapie. «Catharsis », « interprétation », «persuasion » et « analyse » devinrent les nouveaux termes en vogue. Jacqueline Carroy note que dans la littérature hypnotique, la « suggestion » fonctionne comme un terme générique hétérodoxe qui, avec la «suggestion impérative », englobe les injonctions paradoxales et les interprétations ${ }^{6}$. De la même façon, si 1'on examine les études de cas psychanalytiques et psychothérapeutiques du vingtième siècle, $1^{\text {‘on }} \mathrm{s}$ 'aperçoit que le terme « interprétation » fonctionne de façon similaire. Tandis que la formulation théorique des pratiques changeait considérablement, tel n'était pas le cas des pratiques elles-mêmes. Ainsi, sous la rubrique de l'interprétation dans la littérature psychanalytique, il n'était pas rare de trouver des exemples flagrants de directives autoritaires. Freud revendiqua l'absence de suggestion dans la pratique de la psychanalyse. En 1913, Jung déclara que ceci était tout simplement impossible : «Il est impensable

\footnotetext{
${ }^{4}$ C. G. Jung, Versuch einer Darstellung der psychoanalytischen Theorie, Düsseldorf, 1995, Gesammelte Werke, 4, § 433. La Théorie psychanalytique, Paris, Montaigne, 1932.

5 Ibid., §434.

6 Jacqueline Carroy, Hypnose, suggestion et psychologie: l'invention de sujets, Paris, PUF, 1991, p. 179-200.
} 
pour l'esprit critique que la suggestibilité et la suggestion puissent être évitées dans la méthode cathartique. Elles sont présentes partout... même avec Dubois et les psychanalystes qui croient tous travailler de manière purement rationnelle. Aucune technique et aucun auto-effacement ne peuvent aider dans ce domaine ; le médecin travaille, nolens volens, et par-dessus tout peut-être, à travers sa personnalité, c'est-à-dire de manière suggestive'. »

Le comportement du thérapeute devenait inévitablement un modèle pour le patient. Dans l'impossibilité d'y échapper, la seule solution consistait pour lui à atteindre une assez grande connaissance de soi. Jung écrivit : «A de nombreuses reprises, j'ai eu l'occasion de voir que l'analyste arrive toujours dans sa thérapie aussi loin qu'il est parvenu dans son propre développement mo$\mathrm{ral}^{8} . »$

Le but, cependant, n'était pas de diriger le patient, mais de l'inciter à parvenir à la maîtrise et à la connaissance de lui-même. Nous verrons de quelle façon ces questions furent mises en œuvre dans la pratique de Jung.

A partir de 1913, Jung s'engagea dans un processus d'auto-expérimentation qu'il nomma sa « confrontation avec l'inconscient ». La tentative de trouver son propre « mythe » comme remède à une modernité laïque singulièrement dénuée de mythe était au cœur de ce projet. Ceci prit la forme d'une série provocante de fantasmes éveillés de grande ampleur. Il élabora, illustra et commenta ces fantasmes dans une œuvre qu'il nomma Liber Novus ou Livre Rouge et qui se trouve au centre de son œuvre tardive ${ }^{9}$. Il y décrit le processus à travers lequel il retrouva son âme et surmonta l'aliénation spirituelle génératrice du malaise contemporain ; c'est en permettant la renaissance en son âme d'une nouvelle image de Dieu et en développant une nouvelle vision du monde sous la forme d'une cosmogonie psychologique et théologique, qu'il y parviendra. Le Liber Novus représente le prototype du processus d'individuation selon Jung, qu'il tenait pour la forme universelle du

\footnotetext{
${ }^{7}$ C. G. Jung, une correspondance entre C.G. Jung et R. Löy, $G W \S 584$. Sur la vision de jung de l'hypnose et la suggestion, voir mon "'The magical method that works in the dark': C. G. Jung, hypnosis and suggestion", Journal of Jungian Practice and Theory, 3, 2001, p. 5-18.

${ }^{8}$ Ibid., $\$ 587$.

${ }^{9}$ C. G. Jung, Le Livre Rouge Liber Novus, Paris, L'Iconoclaste/ La Compagnie du Livre Rouge, 2011.
} 
développement psychologique individuel.

Il existe peu de récits de ce qui se déroulait dans le cabinet de Jung durant cette période cru-

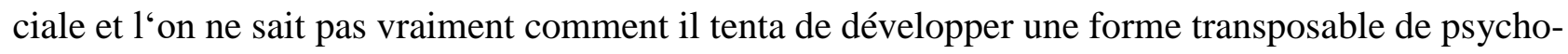
thérapie à partir de son auto-expérimentation.

Rétrospectivement, Jung affirma qu'après sa rupture avec Freud, il avait éprouvé le besoin de développer une attitude nouvelle envers ses patients : «Je décidai pour le moment d'attendre sans préjugé ce qu'ils raconteraient d'eux-mêmes. Je me mis en quelque sorte à l'écoute de ce que le hasard apportait... Il apparut bientôt qu'ils racontaient spontanément leurs rêves et leurs imaginations et je posais simplement quelques questions comme «Qu'est-ce que cela évoque pour vous ?» ou «Comment voyez-vous cela, comment le comprenez-vous ? D'où cela vient-il ? » Des réponses et des associations que fournissaient mes malades, les interprétations découlaient comme d'ellesmêmes. Je laissais de côté tout ce qui était perspective théorique et j'aidais simplement les patients à comprendre leurs images par eux-mêmes. ${ }^{10}$ »

Ceci suggère que la pratique de Jung avec ses patients suivait des procédés semblables à ceux qu'il utilisait pour tenter de comprendre ses propres rêves et visions à cette époque, à savoir la mise en suspens de tout présupposé théorique afin de permettre aux images et figures de s'expliquer d'elles-mêmes. Sa motivation première pour entreprendre son exploration personnelle, déclara-t-il, était d'aider ses patients. Il ne pouvait attendre d'eux quelque chose qu'il n'osait faire lui-même. Il émit un autre commentaire concernant son travail clinique à cette époque : « «Mais la famille et la conscience que j'avais un diplôme de médecin, que je devais secourir mes malades, que j'avais une femme et cinq enfants, et que j'habitais Seestrasse 228 à Küsnacht - c'étaient là des réalités qui me sollicitaient et s'imposaient à moi [...] ma famille et ma profession furent toujours une réalité dis-

\footnotetext{
${ }^{10}$ C. G. Jung, Ma vie. Souvenirs, rêves et pensées, Paris, Gallimard, 1973, p. 198.
} 
pensatrice de bonheur et la garantie que j'existais normalement et réellement ${ }^{11}$. »

Ici, Jung énonce clairement ce que ses patients faisaient pour lui : le convaincre de sa normalité. L'impression générale qui en découle est que sa pratique clinique n'était pas sérieusement affectée par le tumulte qui l'habitait à cette époque.

À l'automne 1911, le psychanalyste et neurologue américain James Jackson Putnam envoya sa cousine Fanny Bowditch Katz en analyse chez Jung. À la mort de son père, celle-ci avait sombré dans une longue dépression. Katz envoya des lettres à Putnam détaillant le déroulement de l'analyse et Putnam lui répondit par des conseils. Il est aujourd'hui courant que les thérapeutes soient supervisés, mais il semblerait que Katz ait été une des premières patientes supervisées. Jung approuvait ce procédé et Katz lisait souvent à Jung les lettres qu'elle écrivait à Putnam, afin de recueillir son approbation. Lorsque Jung rencontra Putnam, il discuta de la situation avec lui. La famille de Katz semblait plutôt méfiante à l'égard de Jung. Le 10 décembre 1912, Putnam écrivit à Katz : « Je suppose qu'il ne faut pas s'étonner que votre charmante tante... trouve les idées du Dr. Jung étranges et répréhensibles. Vous savez, j'imagine, que même la majorité des médecins sont très critiques à propos de toute cette affaire ${ }^{12}$. » En même temps, Putnam adressait un conseil à Jung par l'intermédiaire de Katz: «Dis au Dr. Jung que tu veux te sentir bien et forte et qu'il doit trouver les moyens de t'aider ${ }^{13}$. » Putnam faisait part à Katz de sa propre analyse de Jung : «Un défaut du Dr. Jung (entre nous) est d'être trop péremptoire et je le soupçonne de manquer de certaines formes nécessaires d'imagination et d'être, en fait, une personnalité forte mais vaniteuse, qui peut faire et fait beaucoup de bien, mais qui pourrait aussi avoir tendance à écraser un patient. Il y a à apprendre de lui, mais il ne faut pas le suivre trop aveuglément ${ }^{14}$. »

«Je soupçonne que les méthodes très magistrales du Dr. Jung sont susceptibles d'affecter quelques-uns de ses patients bien plus fortement qu'il ne le réalise ; tu ne dois pas devenir dépen-

\footnotetext{
${ }^{11}$ Ibid., p. 220-221.

${ }^{12}$ Putnam à Bowditch Katz, 10 décembre 1912, Katz papers, Countway Library of Medicine.

${ }^{13}$ Putnam à Bowditch Katz, 12 décembre 1912.

${ }^{14}$ Putnam à Bowditch Katz, 7 janvier 1912.
} 
dante de lui, ni hésiter à formuler en pensée des jugements critiques à son égard ${ }^{15}$. » «Je ne peux que soupçonner que tu souffres en partie de l'influence du Dr. Jung et de sa tendance à prendre les choses de façon excessivement personnelle. Peut-être que je me trompe, mais il n'y a aucun mal à réaliser qu'il n'est pas non plus Dieu, mais un homme aveugle essayant de conduire les aveugles, et que tu es tout aussi libre de le critiquer qu'il ne ''est $^{16}$. »

Putnam rapporta le récit par Katz de son analyse avec Jung à Ernest Jones, qui se montrait extrêmement critique vis-à-vis des procédés de Jung. L'indiscrétion était alors la règle fondamentale de la politique psychanalytique ${ }^{17}$. «Jung ne semblait pas soucieux de maintenir une stricte confidentialité. Au Burghölzli, il était courant que les psychiatres correspondent avec les familles des patients. Dans le cas de Bowditch Katz, Jung fit de même. Il écrivait à la mère de Katz en lui faisant part de ses progrès. En 1915, il l'informa que sa fille devait en passer par un processus de maturation avant d'atteindre l'état de pleine indépendance nécessaire à sa bonne santé. Il ajouta qu'il lui était impossible de lui décrire ce processus car cela nécessiterait un gros ouvrage ${ }^{18}$. »

De plus, Jung n'était pas limité par les séances de cinquante minutes. Ainsi par exemple, à l'époque où il effectuait son service militaire au Château d'Oex, il s'arrangea pour rencontrer Katz à la gare pour une séance qui dura deux heures et demie ${ }^{19}$. James Kirsch, qui fut analysé par Jung à la fin des années vingt, rappelle que certains de ses entretiens avec lui se déroulaient au cours de promenades dans les collines près de Küssnacht ${ }^{20}$. Durant la période estivale, Jung consultait parfois dans son jardin.

En cours d'analyse avec Katz, Jung l'envoya simultanément en analyse chez son assistante Maria Moltzer ${ }^{21}$. Au début de l'année 1913, Moltzer et Toni Wolff avaient été admises comme

\footnotetext{
${ }^{15}$ Putnam à Bowditch Katz, 10 décembre 1912.

${ }^{16}$ Putnam à Bowditch Katz, 10 dcembre 1913.

${ }^{17}$ Putnam cite à Bowditch Katz une réponse de Jones.

18 Jung à Madame Katz, 27 février 1912.

19 Jung à Bowditch Katz, 11 janvier 1915.

${ }^{20}$ James Kirsch, entretien avec Gene Nameche, 1968, Jung biographical archive, Countway Library of Medicine.

${ }^{21}$ Sur les analyses de Bowdith Katz, voir aussi E. Taylor, “C. G. Jung and the Boston psychopathologists 1902-1912”, Carl Jung and Soul Psychology, édité par E. Mark Stern, New York, Routledge, 1986, p. 131-144. Sur Bowditch Katz,
} 
membres en apprentissage de la société psychanalytique de Zurich ${ }^{22}$. Lorsque Moltzer commença à exercer, elle était supervisée par Jung. Voici comment ce dernier décrivait sa supervision au psychiatre américain Smith Elly Jeliffe : « Je lui confiai entièrement les cas à la seule condition qu'en cas de difficulté, elle me consulte ou m'envoie le patient en vue d'un contrôle de ma part. Mais un tel arrangement n'exista qu'au début. Par la suite, Mme M. Moltzer exerça assez indépendamment et efficacement. Financièrement, elle était indépendante, étant payée directement par ses patients... j'instituai des réunions hebdomadaires avec mon assistante, au cours desquelles tout était soigneusement établi sur une base analytique ${ }^{23}$. »

La pratique de l'analyse en tandem allait devenir une spécificité de la technique classique jungienne ; il était considéré comme souhaitable pour une personne d'être analysée par un homme et par une femme.

L'auto-exploration de Jung consistait à induire des fantasmes éveillés et à s'y engager, à entamer le dialogue avec les personnages qui surgissaient et à dessiner et peindre les images qui apparaissaient $^{24}$. Il suggérait à ses patients de se livrer à de telles pratiques. En 1916, il décrivit ces procédés dans un article non publié, « La fonction transcendante ${ }^{25} »$. En effet, cet article montre comment, à partir de son auto-expérimentation, Jung tenta de développer une méthode psychothérapeutique généralisable qu'il nommera par la suite « imagination active ». Il notait qu'il s'agissait de commencer par se concentrer sur un état particulier et d'essayer de devenir le plus conscient possible des fantasmes et associations s'y rapportant. Le but étant de laisser libre cours à la fantaisie par un processus de libre association, sans pour autant s'éloigner de l'affect initial. Ce qui conduisait à une expression concrète ou symbolique de l'état ou de l'humeur en question, avec pour effet

Moltzer et Jung, voir mon Cult Fictions: C. G. Jung and the Founding of Analytical Psychology, Londres, Routledge, 1998.

2230 janvier 1913, Protokolle des Psychoanalytischen Vereins, archives of the Psychological Club, Zürich.

${ }^{23}$ Jung à Jelliffe, juillet 1915, dans J. C. Burnham and W. McGuire, Jelliffe: American Psychoanalyst and Physcian \& His Correspondence with Sigmund Freud and C. G. Jung, Chicago, 1983, p. 198.

${ }^{24}$ Sur l'évolution des techniques d'auto expérimentation de Jung, voir mon "Jung's practice of the image", Journal of Sandplay Therapy, 24, 2015, p. 7-22.

${ }^{25}$ C. G. Jung, 'Die Transzendente Funktion,' GW 8, § 170-1,. « La fonction transcendante », L'Ame et le Soi, Paris, Albin Michel, 1990. 
de rendre l'affect plus accessible à la conscience et donc plus susceptible d'être appréhendé et compris. Le fait même de se livrer à ce processus était potentiellement générateur de vitalité. Les individus pouvaient écrire, dessiner, peindre ou sculpter, suivant leurs sensibilités. Une fois ces fantasmes produits et incarnés, deux approches étaient possibles : la formulation créative et la compréhension. Les deux étaient interdépendantes et nécessaires à l'émergence de la fonction transcendante. Celle-ci était issue de l'union de contenus conscients et inconscients et produisait un élargissement de la conscience.

À cette époque, Jung encourageait ses patients à s'engager dans des processus similaires d'auto-investigation. Durant ses séances avec Katz, Moltzer parlait ouvertement de ses propres expériences et instruisait Katz des nouvelles conceptions de Jung. Au cours d'une séance, Katz nota dans son journal que : «Parlant de Dieu, [Moltzer] parlait de la conception du Dr Jung d'Abraxas, l'Urlibido à laquelle elle adhère également ; utilisant indifféremment les termes Libido et Horme pour désigner la force individuelle. L'Abraxas est la force cosmique derrière chaque Dieu (le Dieu embrassant le diable est double). Abraxas, une conception monothéiste - le seul pouvoir. Très difficile à comprendre et peu retenu ${ }^{26}$. »

Il n'est guère surprenant que Katz ait trouvé cela difficile à comprendre car il apparaît qu'au cours de cette séance, Moltzer lui avait livré un condensé des Septem Sermones ad Mortuos. Tout comme Jung, Moltzer possédait un livre, dans lequel elle écrivait et peignait. Elle l'appelait sa Bible et encourageait Katz à en faire autant. Cette dernière écrivait dans son journal : «Chacun doit écrire sa bible et en travaillant à la mienne, je trouverai mon adaptation à R. [Rudolf Katz, son mari $]^{27}$ ». Moltzer pensait que l'inconscient ne se constellait pour chacun qu'à travers l'art ${ }^{28}$. Katz finit par rentrer en Amérique et vécut jusqu'à l'âge de quatre-vingt-treize ans. En 1956, elle fit savoir à Jung qu'elle devait son exceptionnel état de santé ainsi que l'originalité de ses bijoux en argent, à ses an-

\footnotetext{
${ }^{26}$ Bowditch Katz, août 1917, Journal, Katz papers.

2717 août 1917 , Ibid.

287 mai, 1917, Ibid.
} 
nées zurichoises ${ }^{29}$.

D’après les notes de Bowditch Katz concernant son analyse avec Moltzer, celle-ci pouvait en partie être considérée comme une analyse avec Jung «par relais », étant donné que Moltzer cherchait à transmettre à Bowditch Katz les nouvelles conceptions de Jung qu'elle avait fait siennes.

En 1912, Tina Keller avait été envoyée en consultation chez Jung par son mari Adolph Keller, membre du cercle des proches de Jung et l'un des premiers pasteurs à s'intéresser à la psychanalyse. Pasteur de l'Église Saint-Pierre de Zurich, il trouvait dans la psychanalyse des outils précieux au conseil pastoral. Durant son enfance, Tina Keller avait souffert de troubles anxieux et ceux-ci avaient resurgi dans sa vie de femme mariée, bien qu'il se fût agi d'un mariage heureux. Adolph Keller demanda conseil à Jung et celui-ci recommanda une analyse. Tina Keller commença son analyse avec Maria Moltzer. Suite à un rêve, Moltzer l'adressa à Jung ${ }^{30}$. Elle se souvient que Jung lui dit : «C'est une grande chance pour vous d'arriver à la psychanalyse à présent que les idées freudiennes ont connu un élargissement ». Et Tina Keller d'ajouter ; «Je suis sûre que je ne serais pas restée en analyse freudienne ${ }^{31} »$. Voici comment elle décrit sa situation : «La préoccupation de mon mari était que je sois libérée de la peur, mais le Dr Jung savait qu'il ne pouvait faire disparaître mes peurs. Il me le dit et ajouta que la peur et l'anxiété n'étaient que des symptômes, que je vivais un "processus d'individuation" et que les symptômes ne diminueraient qu'au fil de l'individuation [...] Le Dr Jung mettait ma foi au défi, il essayait de faire émerger mes doutes inconscients [...] Il était persuadé que l'individu moderne devait parvenir à une expérience religieuse personnelle, et qu'une telle expérience ne peut survenir que lorsque l'on n'a plus rien à quoi se raccrocher $^{32}$. »

\footnotetext{
${ }^{29}$ Fanny Bowditch Katz à Jung, 17 janvier 1956, Jung archive, Swiss Federal Institute of Technology, Zürich.

${ }^{30}$ The Memoir of Tina Keller-Jenny: A Lifelong Confrontation with the Psychology of C. G. Jung, édité par. W. Swan, préfacé par S. Shamdasani, New Orleans, 2011, p. 17.

${ }^{31}$ Ibid., p. 19.

${ }^{32}$ T. Keller, “C. G. Jung: Some memories and reflections”, Inward Light 35, 1972, p. 5.
} 
Il découle de ceci que Jung concevait la tâche de la psychanalyse comme allant largement au-delà de la disparition des symptômes : il s'agissait d'œuvrer à un développement plus élevé de la personnalité. Pour rendre cela possible, l'individu devait accéder à une expérience directe du religieux. Rien n'était plus éloigné de l'athéisme virulent de Freud.

Jung ne faisait pas qu'inciter ses patients à parler spontanément de leurs expériences, il leur rapportait spontanément les siennes. Les patients semblaient tout à fait conscients de ce que Jung traversait. Keller se souvient : «A l'époque où j’étais en analyse avec le Dr. Jung, celui-ci était encore fortement impressionné par cette période d'irruption de l'inconscient [...] C'était pendant la première Guerre Mondiale et le Dr. Jung faisait parfois allusion à ses expériences les plus saisissantes. Une fois, il dit qu'elles avaient fait virer ses cheveux au gris [...] Il parlait souvent de lui et de ses propres expériences ${ }^{33}$.»

Jung ne cachait pas à ses patients le travail créatif en quoi consistait son autoexpérimentation en cours : «Souvent, en cette période des débuts, lorsque l'on arrivait pour l'heure analytique, ledit Livre Rouge était là, ouvert sur un chevalet. Le Dr Jung y avait peint ou y achevait tout juste une image. Parfois, il me montrait son ouvrage et le commentait. Le soin et la précision qu'il apportait à ces images ainsi qu'au texte enluminé qui les accompagnait, témoignaient de l'importance de son entreprise. Le maître montrait ainsi à l'élève que le développement psychique mérite que l'on y consacre du temps et des efforts ${ }^{34}$. »

Une fois, Jung lui montra une peinture dans le Livre Rouge qu'il mit en rapport avec ses liens à sa femme et à Toni Wolf. Keller se souvient : « [Jung] dit, "regardez ces trois serpents entremêlés. C'est ainsi que nous luttons tous les trois avec ce problème'. Ce que je peux dire est qu'il me semblait très important que, même s'agissant d'un phénomène passager, trois personnes accep-

\footnotetext{
${ }^{33}$ T. Keller, Recollections of my encounter with Dr. Jung, Jung biographical archive, Countway Library of Medicine, p. A2.

${ }^{34}$ The Memoir of Tina Keller-Jenny, op cit., p. 21.
} 
tent un destin qui ne se borne pas à leur satisfaction personnelle ${ }^{35}$. »

Lorsque le processus psychique du thérapeute était susceptible de concerner le patient, Jung n'avait aucune réticence à le partager ouvertement. Keller écrit : «L'on se sentait admis au sein d'une atmosphère très spéciale de découverte du monde intérieur et de son mystère [...] À chaque fois que le Dr. Jung parlait de ces expériences, je percevais son émotion. Arriver en séance à ces moments-là, c'était pénétrer dans une atmosphère très spéciale. On sentait le Dr. Jung extrêmement attentif aux fragments qui "lui parvenaient" et qu'il devait tenter de comprendre, mais qui étaient clairement au-delà de ce que le cerveau humain peut saisir. Tout était fluide, ce qu'il disait était hésitant, paradoxal et plein de contradictions apparentes ${ }^{36}$. »

Jung développa un ensemble de principes spécifiques qu'il exhortait ses patients à mettre en pratique. Keller écrit : «Le Dr. Jung insistait sur la préparation. Il nous prescrivait d'écrire nos rêves et l'association à chacun de ses éléments [...] La technique la plus importante que j'aie apprise au cours des séances avec le Dr. Jung était celle qui consistait à écrire "à partir de l'inconscient." Très tôt dans mon analyse, le Dr. Jung dit, "Vous devez dès à présent commencer à vous préparer pour le temps où vous ne viendrez plus me voir. À chaque fois que vous partez d'ici, y compris en descendant les escaliers, vous avez encore des questions. Écrivez-les comme si c'étaient des lettres que vous m'adressiez. Vous n'avez pas besoin d'envoyer ces lettres. Si vous posez une question, dans la mesure où vous voulez véritablement une réponse et que vous n'ayez pas peur de cette réponse, elle existe tout au fond de vous. Laissez-là émerger.” J'essayai et rien ne venait, je le dis au Dr. Jung. Mais il insistait. Il dit même, "Vous savez sûrement $\operatorname{prier}^{37}$ !". »

Le rôle de la prière comme l'une des sources de la technique analytique de Jung n'a guère été

\footnotetext{
${ }^{35}$ Entretien de Tina Keller avec Gene Nameche, 1969, R. D. Laing papers, University of Glasgow, p. 27.

${ }^{36}$ T. Keller, Recollections of my encounter with Dr. Jung, op. cit., p. B12, A2.

${ }^{37}$ The Memoir of Tina Keller-Jenny, op. cit., p. 23.
} 
commenté. La psychologie de la prière constituait pourtant un volet important de la psychologie des religions, et elle était également présente dans les recherches sur le psychisme. La nouvelle psychologie de la suggestion, l'auto-suggestion ainsi que la télépathie, étaient invoquées pour expliquer la prière. Frederic Myers tenta d'avancer une définition spirituelle mais non-théologique de la prière ou de la supplication, définie dans ses grandes lignes comme un appel à l'invisible. Il s'agissait selon lui d' « une tentative d'obtenir un effet bienfaisant de la part d'êtres invisibles au moyen d'une disposition intérieure de notre esprit ${ }^{38} \gg$. À en juger par la description que fait Keller des recommandations de Jung, il est ici question d'une forme de prière non-dénominationnelle. L'inconscient est l'invisible, le pouvoir supérieur auquel l'on fait appel pour en être instruit et guéri.

L’intérêt de Jung pour la prière est présent dans ses imaginations actives de début 1914. Dans celle du premier janvier 1914, le Je de Jung se retrouvait dans une vallée déserte où il rencontra un anachorète nommé Ammonius ${ }^{39}$. Ce dernier l'enjoignit ne pas oublier sa prière du matin. Je réalisa alors que nous avions perdu notre sens de la prière. Celle du 14 janvier de la même année rapporte que Je cherchait à emprunter un exemplaire de l'Imitation du Christ, dans le but de «prier ou quelque chose d'approchant » car à certains moments, la science nous rendait malades ${ }^{40}$.

En même temps, Jung s'abstenait de préconiser la prière traditionnelle au cours des séances. Kurt Binswanger se souvient : «Maeder croyait bon de prier avec ses patients durant les séances. Chose à laquelle Jung ne pouvait adhérer ${ }^{41} »$.

Au décours de l'analyse, Tina Keller ressentit tour à tour de l'amour et de la haine envers Jung. «Le Dr. Jung ne parlait jamais de "transfert," mais il m'obligeait à affronter le fait que j'étais “amoureuse.” Il eût été plus simple d'utiliser un terme technique. La théorie du Dr. Jung était que j'étais “amoureuse” d'une certaine qualité (ou archétype) qu'il représentait, et qui avait touché ma

\footnotetext{
${ }^{38}$ F. Myers, Human Personality and its Survival after Bodily Death, London, 1903, vol. 2, p. 310.

${ }^{39}$ C. G. Jung, The Red Book. Liber Novus, p. 269. Le Livre Rouge Liber Novus, op. cit.

${ }^{40}$ Ibid., p. 292.

${ }^{41}$ Kurt Binswanger, entretien avec Gene Nameche, Jung biographical archive, Countway Library of Medicine, p. 14.
} 
psyché. Si et dans la mesure où je serais capable de reconnaître cette qualité ou cet élément inconnu en moi-même, je serais alors libérée de lui en tant que personne ${ }^{42}$. »

Non content de se dispenser de langage technique pour décrire la relation de son patient avec lui, Jung semble en avoir fait autant concernant ses relations avec ses patients, leur parlant assez ouvertement de ce qu'il ressentait. Keller écrit : «Il était convaincu de la signification d'une telle manifestation, et disait que ce que j'avais apporté était une telle ouverture qu'il me devait une certaine valeur spirituelle qui fertiliserait ma psyché, et que mon "individuation" serait un "enfant spirituel.” Cela sonnait bien. Il le pensait sincèrement, mais cela ne s'est pas avéré vrai ${ }^{43}$. »

Ici, Jung avoue ouvertement le rôle actif du thérapeute dans la rencontre thérapeutique, fertilisant la psyché du patient, donnant naissance à son individuation. Après son analyse avec Jung, Tina Keller entreprit une analyse avec Toni Wolff.

Loin d'être solitaire, la confrontation de Jung à l'inconscient était une entreprise collective, dans laquelle il entraînait ses patients. Autour de Jung, s'était formé un groupe avant-gardiste engagé dans une expérimentation sociale dont tous espéraient qu'elle transformerait leurs vies et celles de leur entourage ${ }^{44}$. Keller écrit : «Durant la Première Guerre Mondiale, en plein sentiment de catastrophe, alors que les valeurs culturelles s'effondraient, alors que prédominaient la consternation et la désillusion générales, un petit groupe autour de Jung participait à sa vision d'un monde intérieur en déploiement. Nombre d'entre nous fûmes déçus par la suite. La vision était trop vaste et regardait vers le futur ${ }^{45}$.»

Tina Keller devint psychothérapeute. Durant des décennies, elle fut la principale représen-

\footnotetext{
${ }^{42}$ T. Keller, Recollections of my encounter with Dr. Jung, op. cit., B19.

${ }^{4}$ Ibid.

${ }^{44}$ Pour l'étude d'un autre cas important de cette époque, voir V. de Moura,"Learning from the patient: the East, synchronicity and transference in the history of an unknown case of C.G. Jung", Journal of Analytical Psychology, 59, 2014, p. 391-409.

${ }^{45}$ T. Keller, "Recollections of my encounter with Dr. Jung", o. cit., A5.
} 
tante de Jung à Genève. Elle s'adressait à Jung pour des supervisions. À une certaine occasion, elle évoqua le cas d'un patient borderline. Jung lui dit : «Vous n'avez pas le droit d'expérimenter comme je le fais car j'ai un nom à présent. S'il vous arrive quelque chose, c'est différent que si c'est à moi que cela arrive ${ }^{46}$. » Jung était tout à fait conscient de la nature expérimentale de sa pratique et de la protection que lui octroyaient son statut et sa qualité de médecin. Emil Medtner, un autre de ses patients durant cette période, affirma que s'il n'eut été Jung, il aurait partagé le destin de Nietzsche et serait « devenu fou ». Il assimilait l'analyse à ce que Goethe avait qualifié de « cure psychique où l'insanité est convoquée pour guérir l'insanité ${ }^{47}{ }^{\text {». }}$

En 1913, Edith Rockfeller McCormick et son mari se rendirent à Zurich pour faire une analyse avec Jung. Les McCormick envoyaient des lettres au père d'Edith, John. D. Rockfeller, faisant état de leurs progrès et exprimant leur profonde admiration pour Jung. Le 15 juin 1915, Harold McCormick lui écrivit : «Ce n'est pas ici un tabernacle de la joie mais un sanctuaire où ceux qui cherchent ne s'adressent qu'à eux-mêmes. C'est dans cet esprit que j'ai encore différé mon départ et qu'Edith se trouve encore retenue. Pour chacun d'entre nous, chaque jour compte. Ce n'est pas un lieu (l'école de Zurich) où l'on est encouragé à rester au-delà d'un temps juste ou normal, toute la question étant celle du meilleur degré. Car quiconque s'intéresse vraiment à la psychologie analytique et y trouve de l'aide ne songe nullement à la laisser de côté. Parce que s'il y a bien quelque chose, c'est de la vivre. Et plus on étudie, mieux on est préparé à vivre selon ses bases. Ainsi faut-il que chacun s'en retourne dans la vie, faute de quoi elle (la psychologie analytique) faillirait à son propos. L'idée fondamentale est de s'enseigner soi-même. Il n'est pas toujours facile, et cela est rendu encore plus difficile du fait des résistances conscientes, de suivre le chemin lorsqu'on l'a soi-même tracé. Mais nous avons une tendance naturelle, dont nous avons à nous défendre, à préférer la quiétude de la vie ici à l'adversité et aux difficultés de vivre et de la vie en général. Ni Edith ni moi n'en sommes encore à ce point et nul doute que lorsque nous l'aurons atteint, nous

\footnotetext{
${ }^{46}$ T. Keller, entretien avec Gene Nameche, op. cit., p.18.

${ }^{47}$ Cité dans M. Ljunggren, The Russian Mephisto: A Study of the Life of Emilii Medtner, Stockholm, 1994, p. 91
} 
saurons l'assumer ${ }^{48}$. »

On comprend à la lecture que pour les McCormicks, comme pour un bon nombre de patients durant cette période, l'analyse devenait non seulement une forme de thérapie mais la base d'un nouveau mode de vie ancré dans la psychologie.

La première phase de l'auto-expérimentation de Jung avait consisté en un « retour » à luimême, une reconnexion avec son âme. D'après les lettres de Harold McCormick, il apparaît que Jung avait réussi à aider certains de ses patients à en faire autant. Le premier septembre 1915, H. Mc Cormick écrit à John.D.Rockfeller : « Nous faisons de notre mieux et apprécions grandement cette occasion de travailler sous la belle inspiration et la conduite du Dr Jung - nous incitant à nous montrer tels qu'en nous-mêmes et à nous permettre de mieux nous connaître. Cela peut sembler banal mais je vous dis le plus sincèrement du monde ma surprise de constater combien peu je me suis connu jusqu'ici et le peu de cas que j'ai accordé à faire connaissance, à me fréquenter et à entrer en intimité avec moi-même. On me dit qu'il y a de grandes possibilités ( richesses ?) de ce côté-là, sans aucunement donner dans l'auto-adulation ${ }^{49}$. »

Leur fils, Fowler McCormik, fit également une analyse avec Jung durant l'hiver 1916-1917. Il se souvient que durant de nombreuses séances, «Jung était occupé à sculpter du bois tandis que nous parlions ${ }^{50} \gg$.

Nous avons vu que Jung recommandait à ses patients de lui écrire des lettres sans les lui envoyer. J'ai trouvé par hasard plusieurs patients qui faisaient de la sorte ${ }^{51}$. Ce qui suit est extrait d'une lettre de Cary de Angulo et nous donne des indications supplémentaires quant à la façon dont Jung maniait le rapport avec ses patients. Cary de Angulo (née Fink) faisait partie de la première génération de femmes à avoir obtenu le titre de médecin aux États-Unis, bien qu'elle n'eût jamais

\footnotetext{
${ }^{48}$ Harold McCormick à John D. Rockefeller, 15 juin 1915, Rockefeller archives center, Terrytown, NY.

${ }^{49}$ Harold McCormick à John D. Rockefeller, 1 septembre 1915, Rockefeller archives center.

${ }^{50}$ Fowler McCormick entretien avec Gene Nameche, Jung biographical archive, Countway Library of Medicine, op. cit., p. 8.

${ }^{51}$ Ainsi par exemple Rivkah Scharf, (je remercie Nomi Kluger Nash de m'avoir donné accès à ces documents).
} 
exercé. Suite à l'échec de son mariage avec le brillant linguiste et ethnologue Jaime de Angulo, elle se rendit à Zurich en 1921 pour travailler avec Jung. Voici ce qu'elle écrit au sujet de leur relation thérapeutique : «Le fait essentiel est qu'en errant dans l'univers complètement détachée, je vous ai rencontré et suis entrée dans une union indissoluble avec vous. Cela s'est fait automatiquement sans que je le veuille ou pas, juste parce que vous êtes ce que vous êtes. À un tel "vous” je peux écrire, car ce "vous" me donne une place dans votre vie, une place unique de grande intimité et pourtant d'extrême retenue. C'est ainsi que je définis une relation symbolique. Je suis tout à la fois à l'intérieur de votre être, et complètement, à jamais séparée de vous [...] Chaque heure que je passe avec vous est sacrée pour moi, non parce que je vous vénère, mais parce que je me rapproche ce faisant de certaines valeurs que vous exprimez plus manifestement que quiconque ${ }^{52}$. »

Cary de Angulo était plus réceptive que Tina Keller à ce que Jung proposait. Elle décrit avec sensibilité la sincérité de son entreprise et comment pour elle, il s'agissait de retrouver le sens des valeurs religieuses plutôt que de vénérer Jung. Ce dernier appréciait son intelligence et son jugement et il lui demanda conseil concernant le Livre Rouge. Elle note : «Vous avez fait un rêve la nuit dernière dans lequel j'apparaissais déguisée et je devais travailler au Livre Rouge, vous y aviez pensé toute la journée et surtout durant l'heure du Dr Wharton précédant la mienne ( très plaisant pour elle je dois dire...). Ainsi que vous l'aviez dit, vous étiez décidé à me faire partager tout votre matériel inconscient représenté par le Livre Rouge, etc. afin de voir ce qu'un observateur étranger et impartial en dirait. Vous pensiez que j'avais un bon sens critique, impartial... Pour vous-même, vous disiez avoir toujours su que faire de vos idées mais dans ce cas, vous étiez perplexe. À chaque fois que vous les approchiez, vous étiez emmêlé et n'étiez plus sûr de rien. Vous aviez la certitude que certaines d'entre elles étaient d'une importance majeure mais ne parveniez pas à en trouver la forme appropriée - en l'état où elles étaient, disiez-vous, elles auraient pu tout autant provenir droit d'une maison de fous ${ }^{53}$. »

\footnotetext{
${ }^{52}$ Cary Baynes (née de Angulo), 14 janvier 1923, letter drafts, Baynes papers, Contemporary Medical Archives, Wellcome Library, London.

${ }_{53} 26$ janvier 1924, Ibid.
} 
Jung lui demanda de transcrire le Livre Rouge. Il considérait que la tâche comportait également une valeur pédagogique. Elle nota qu'il lui expliquait « les choses à mesure que j'avançais... De cette façon, nous en venions à discuter de nombreux sujets qui ne se présentaient jamais durant les séances d'analyse et je comprenais vos idées dans leurs fondements ${ }^{54} \gg$.

Jung conduisait également des analyses par correspondance. Jaime de Angulo envoyait ses rêves à son ex-femme. Elle en discutait au cours de ses séances avec Jung et renvoyait à Jaime les interprétations de Jung. Durant une séance en date du 14 février 1924, elle présenta à Jung des notes rédigées par Jaime de Angulo sur lui-même et sur certains rêves qu'il avait envoyés. Le jour suivant, elle lui envoya les commentaires généraux de Jung : «Il dit qu'il chercherait à te « modérer » si tu étais avec lui, ce qu'il ferait en s'assurant que tu aies tout à fait compris les concepts avant de te précipiter sur les processus [...] N'étant pas en mesure de te freiner personnellement, il te suggère de relire très attentivement Psychologie de l'inconscient, ainsi que les Types, en prenant des notes et en commentant les passages que tu ne comprends pas et en m'envoyant ces notes pour les soumettre à la critique ${ }^{55}$. »

Elle continua à lui envoyer l'interprétation de Jung de quatre de ses rêves. Bien que Jung incitât Jaime de Angulo à étudier ses écrits de près, ceci ne constituait pourtant pas une façon systématique de procéder. Dans la même lettre, elle relayait le conseil de Jung concernant un cas suivi par Jaime de Angulo : «Il a dit que tu n'aurais en aucun cas dû tenter de lui expliquer une quelconque théorie, mais que si tu avais l'intention d'entreprendre un travail clinique avec lui, le transfert aurait dû être créé pour préparer le terrain, et la théorie administrée avec de grandes précautions et une grande attention à sa capacité à l'assimiler ${ }^{56}$. »

Les patients en analyse avec Jung écrivaient naturellement à leurs amis en leur relatant leurs expériences à Zurich. Au milieu des années vingt, le créateur de décors de théâtre américain

\footnotetext{
${ }^{54}$ Ibid.

${ }^{55}$ Cary de Angulo à Jaime de Angulo, 15 février 1924, Cary Baynes papers, Ibid.

${ }^{56}$ Ibid.
} 
Robert Edmond Jones vint se faire analyser par Jung. Il décrivit ses expériences à Mabel Dodge :

«Cela fait deux semaines que je travaille avec cet homme et je commence déjà à me mouvoir dans un monde où règnent les visions magiques les plus anciennes d'états d'âme et de commencements du Temps. Ceci n'est pas de la psychanalyse ni aucune chose de la sorte. Cet homme est un homme sage possédé par des secrets [...] Ce travail n'est pas simplement thérapeutique. Il est serein et austère et requiert de la discipline. Il y a beaucoup de Gurdjieff là-dedans. J'aimerais pouvoir te décrire cette expérience mais cela est inutile. C'est en vérité plus ésotérique qu'autre chose, une initiation à l'état d'homme. Il n'y a là aucune trace de médecine (thérapeutique ?). Un voyage mystique subtil et terrible, des tourments, des états de veille, des illuminations. Je crois que nous avons une très bonne alliance de travail. Je me suis avancé de la bonne façon dès le début, sans la retenue de nombreux autres patients; et ce fut un tel soulagement de constater que je n'étais pas homosexuel et voué à être un de ceux-là. Jung dit que j'ai le don le plus remarquable qu'il ait jamais vu d'animer les autres. Le surnom qu'il m'a donné est Briseur de Carapaces parce que j'ai extirpé de leurs carapaces une demi-douzaine de ses patients et que je les ai amenés à le reconnaître. Et il m'a quelquefois appelé Donneur de Vie ${ }^{57}$. »

Dodge recevait également des comptes-rendus de Mary Foote de son analyse avec Jung. Attirée dans le cercle magique, elle envoya à Foote ses propres rêves afin que Jung les analyse ${ }^{58}$. Ces réseaux épistolaires jouèrent un rôle crucial dans la propagation de l'analyse et donnèrent forme aux attentes des futurs patients. Ainsi que Foote en informa Dodge, «Jung ne fait pas disparaître les complexes et il pense que tout progrès provient du conflit, alors je suppose que nous y serons confrontés pour le restant de nos jours ${ }^{59} »$. Les lettres adressées aux proches et aux familiers depuis $\mathrm{Zu}$ rich contribuèrent grandement à promouvoir l'analyse en Amérique et en Angleterre et à la faire exister socialement. À travers ces voies d'échange, les développements dans le monde germanophone se répandirent dans le monde anglophone.

\footnotetext{
${ }^{57}$ Robert Edmond Jones à Mabel Dodge, 1926, Dodge papers, Beinecke library, Yale University.

${ }^{58}$ Mary Foote à Mabel Dodge, novembre 1925, Dodge papers, Ibid. Sur Mary Foote, voir R. Trousdell, "The lives of Mary Foote: painter and Jungian”, Journal of Analytical Psychology, 61, 2016, p. 588-606.

${ }^{59}$ Mary Foote à Mabel Dodge, 1929, ibid.
} 
Les instructions de Jung à ses patients quant à la conduite de l'imagination active étaient très précises. Il racontait ce que lui-même avait expérimenté. En 1926, Christiana Morgan vint en analyse chez Jung. Acquise à ses idées grâce à la lecture des Types Psychologiques, elle s'était tournée vers lui en quête d'aide concernant ses problèmes relationnels et ses dépressions. Dans une séance de 1926, elle note les conseils de Jung sur la production de visions : «Bien, vous voyez qu'elles sont trop floues pour que je puisse en dire grand-chose. Ce n'est que le début. Dans un premier temps, n'utilisez la rétine de votre œil que dans le but d'objectiver. Ensuite, au lieu d'essayer de continuer à forcer l'image, contentez-vous d'y jeter un coup d'œil. Puis, lorsque vous voyez ces images, vous tentez de les saisir et de voir où elles vous emmènent - comment elles changent. Et vous essayez vous-même d'entrer dans l'image - d'en devenir un des acteurs. Quand j'ai commencé à faire cela, j'ai vu des paysages. Ensuite j'ai appris comment me placer dans ces paysages, puis les personnages m'ont parlé et je leur ai répondu ${ }^{60}$. »

Jung décrivait ainsi en détail ses propres expériences avec pour instruction d'en faire autant ; son rôle consistait à superviser ses patients dans l'expérimentation de leur propre flux d'images. Morgan note un propos de Jung : «Il me semble que je dois vous dire quelque chose au sujet de ces fantasmes...ils semblent en l'état plutôt minces et pleins de répétitions de motifs identiques. Il n'y a pas en eux assez de flamme et de chaleur. Ils devraient être plus brûlants...Vous devez y être plus, c'est-à-dire que vous devez y être votre propre conscience critique - imposer vos jugements et vos critiques $^{61}$. »

Jung alla jusqu'à suggérer à ses patients de préparer leur propre Livre Rouge. Morgan rapporte le propos suivant : « Je vous conseille de consigner tout cela de la plus belle manière possible - dans un livre joliment confectionné. Cela vous semblera une façon de banaliser vos visions - c'est justement ce qu'il convient de faire -, ainsi, vous serez libérée de leur pouvoir. Si vous faites cela avec

\footnotetext{
${ }^{60}$ Christiana Morgan, 8 juillet 1926, analysis notebooks, Countway library of Medicine.

${ }^{61}$ Ibid., 12 octobre 1926.
} 
ces yeux par exemple, alors ils cesseront de vous hanter. N'essayez jamais de faire revenir des visions. Pensez-y en imagination, essayez de les peindre. Ainsi, lorsque ces choses seront déposées dans un livre précieux, vous pourrez consulter le livre, en tourner les pages, et elles seront votre église - votre cathédrale-, les lieux silencieux de votre esprit où vous trouverez à vous ressourcer. Si quelqu'un vous dit que cela est morbide ou névrotique et que vous l'écoutiez, alors vous perdrez votre âme, car ce livre contient votre âme ${ }^{62}$. »

Avec son autorisation, Jung utilisa le matériel de C. Morgan au cours d'un séminaire qui s'étendit de 1930 à $1934^{63}$. Celle-ci trouva fort inspirant l'usage qu'il en fit. «Les notes du séminaire sont arrivées et je vous en remercie. Je les ai lues et j'ai refermé le livre avec une prière - un hymne - de gratitude envers vous, de n'avoir point dégradé - voire au contraire mis en valeur -, l'auguste qualité de ces visions. Je souhaite autant que faire se peut, exprimer jusqu'à quel point une telle expérience peut changer une vie - et comment, de fait, cela marche dans la vie actuelle. Comment le sens de la vie est la nécessité de donner corps à ces visions (ou peut-être devrait-on dire agir sous leur impulsion). J'ai particulièrement aimé tout ce que vous avez dit au sujet de la face animale, avec laquelle j'avais perdu la connexion cet hiver ${ }^{64}$. »

Tandis que certains patients adoptaient pleinement les suggestions de Jung, d'autres se montraient plus critiques. En 1919, le psychologue anglais William McDougall entreprit une analyse avec Jung. Durant le déroulement de l'analyse, McDougall, Jung et son assistant Peter Baynes firent de la voile et dînèrent ensemble. Amy Allenby, étudiant de Jung, se rappela plus tard : «Baynes et Jung avaient remarqué que McDougall était fermement retranché derrière sa persona et qu'il était impossible d'accéder à sa vraie personnalité. Alors Jung proposa d'inviter McDougall à une soirée près du lac et de lui servir du vin jusqu'à ce qu'il soit un peu ivre ; c'est ce qu'ils firent et cela se produisit $^{65}$. »

\footnotetext{
62 Ibid., 12 juillet 1926.

${ }^{63}$ C. G. Jung, Visions: Notes of the Seminar Given in 1930-1934, édité par Claire Douglas, Bollingen Series, Princeton, NJ, 1997, 2 volumes.

${ }^{64}$ Morgan à Jung 31 juin 1931, Jung Archive, Swiss Federal Institute of Technology, Zürich.

${ }^{65}$ Amy Allenby, entretien avec Gene Nameche, Jung biographical archive, Countway library of Medicine, p. 3.
} 
D’après les standards contemporains de la psychothérapie, de telles pratiques seraient considérées non professionnelles et comme une «transgression du cadre ». Ce genre de jugement est anachronique. Jung n'a jamais eu une telle conception du cadre. Pour lui, il n'y avait pas de stricte séparation entre l'analyse et la vie. L'analyse était conçue comme devant mener à une reformulation psychologique de la société, à travers l'incitation chez l'individu à forger de nouvelles attitudes et valeurs. Parmi celles-ci, figuraient au premier plan la libération de toute hypocrisie, couplée à l'ouverture d'esprit et à l'honnêteté dans les relations personnelles.

McDougall ne fut guère convaincu par son analyse avec Jung. Peu après, il écrivit dans l'un de ses ouvrages : «Je me suis remis entre les mains du Dr Jung et lui ai demandé d'explorer les profondeurs de mon esprit, mon “inconscient collectif”. Et le résultat a été - “ je suis toujours sorti par la porte par laquelle je suis entré" [...] Il me semble trouver en moi des traces ou des indications des “archétypes" du Dr Jung, mais des traces vagues et douteuses. Peut-être suis-je trop hybride pour avoir des archétypes clairement identifiés ; peut-être mon “inconscient collectif” - si toutefois j'en ai un - est-il emmêlé, confus et trouble ${ }^{66}$. »

En 1926, il publia, An Outline of Abnormal Psychology ${ }^{67}$. Dans son chapitre sur les théories de Jung, il reproduisit certains de ses rêves, l'analyse que Jung en avait fait et ses propres interprétations. Intrigué par ce compte-rendu, Smith Elly Jeliffe demanda à Jung de plus amples informations sur le traitement de McDougall. Jung lui répondit : «J'ignore si je suis tenu à la discrétion dans le cas de McDougall, dans la mesure où il se désigne lui-même comme une personnalité désespérément normale. Je n'ai probablement aucune raison de considérer son cas comme relevant du concept de discrétion médicale. Il n'y a d'ailleurs pas grand-chose au sujet de quoi être indiscret. C'était vraiment comme il le décrit : très peu de rêves apportés au Dr Jung pour en discuter, en retenant si possible toute réaction qui eût pu s'avérer désagréable. Ce furent, ainsi que vous vous en

\footnotetext{
${ }^{66}$ W. McDougall, National Welfare and National Decay, London, 1921, p. 134.

${ }^{67}$ W. McDougall, An Outline of Abnormal Psychology, London, 1923, p. 181-205.
} 
doutez, un nombre très modeste de conversations et rien d'autre qu'un passage à travers les procédures de l'analyse dont, je le crains, le Professeur McDougall ne possède pas la moindre idée. J'apprécie cependant ses expériences avec des rats et n'aurais pas de désaccords à ce sujet. Cependant, j'ai pu constater que les personnes qui sont absolument vierges de psychologie sont en général profondément convaincues de leurs compétences psychologiques ${ }^{68}$. »

Jung insistait pour que ses patients se préparent à l'analyse. Il leur demandait de suivre une analyse avec l'un de ses assistants avant de le voir, et également d'effectuer une supervision en psychologie analytique. Dans les années vingt, Jung demanda à Cary de Angulo de superviser certains de ses patients. Il lui demanda notamment de prendre en supervision, en son absence, l'une de ses patientes, le Dr Bond. Celle-ci selon lui était une « introvertie au dernier degré » et avait besoin d'un important travail préparatoire en termes de connaissances générales ${ }^{69}$.

En plus de la supervision, la préparation prenait la forme d'analyses préalables avec l'un des élèves de Jung. Il écrivit à l'écrivain américain Léonard Bacon que les patients devaient commencer leur analyse avec l'un d'entre eux afin d'être « correctement préparés » avant de le voir ${ }^{70}$.

Le sens d'une telle préparation était de permettre à Jung de se concentrer sur le développement ou l'individuation de ses patients. À partir des années vingt, les individus ne venaient pas vers Jung sans savoir à quoi s'attendre. Ils étaient sélectionnés et préparés. Le rôle social de patient analytique - ici en l'occurrence, de patient de Jung - devait être créé. S'ils avaient d'importants problèmes personnels à résoudre, Jung laissait généralement cela à ses assistants. Ceci indique que ce qui s'ensuivit fut le résultat de procédures plutôt inhabituelles. À propos de sa pratique, Jung notait qu'il avait très peu de nouveaux cas et que la plupart d'entre eux avaient eu une expérience préalable de la psychothérapie. En 1954 il écrivit que, tout comme en chirurgie, il existait une psycho-

\footnotetext{
68 Jung à Jeliffe, 7 juin 1932, dans Burnham and McGuire, Jeliffe: American psychoanalyst and physician \& his correspondance with Sigmund Freud and C. G. Jung, op. cit., p. 326.

69 Jung à Cary de Angulo, 17 août 1925, Baynes papers.

70 Jung à Leonard Bacon, 26 juillet 1926, Bacon papers, Beineke Library, Yale University.
} 
thérapie mineure et majeure, et que c'est cette dernière qui l'intéressait. « Il s'agit d'une minorité de patients ayant des exigences intellectuelles d'un certain niveau, et seuls ceux-là connaissent un développement qui place le médecin face à des problèmes du type qui est décrit ici ${ }^{71}$. »

Dans ses écrits ultérieurs, Jung insista sur le fait que l'individuation de ses patients n'était pas un produit de la suggestion mais un processus naturel spontané, qui se trouvait simplement accéléré par l'analyse. Certains cependant concevaient la chose autrement. Ainsi, Tina Keller se souvient : «Je crois qu'il s'agissait d'une sorte de contagion en raison du processus dynamique dans lequel le Dr. Jung était encore engagé et que ses proches s'identifiaient à lui. Cela pose la question de savoir si un pionnier qui se trouve dans un état "exceptionnel” peut travailler sûrement en tant que thérapeute ? Ou peut-être existe-t-il une qualité spéciale chez un tel homme, stimulante à un tel point pour les personnes privilégiées (de son entourage), que les avantages outrepassent les dangers $^{72} ? \gg$

Durant son analyse avec Jung, Tina Keller fut entraînée avec d'autres dans le processus que Jung traversait. Son expérimentation sur lui-même était simultanément une expérimentation sur eux. En 1929, Jung décrivit explicitement son objectif comme étant de faire atteindre (à son patient) « un état de l'âme où mon patient commence à expérimenter avec son être propre ${ }^{73}$ ». Les interactions respectives jouèrent un rôle crucial dans la constitution de la psychologie analytique comme mouvement social. C'est ainsi que l'auto-expérimentation de Jung généra un effet de transformation sur un nombre croissant d'individus, à mesure que ceux-ci adoptaient ses conceptions et le laissaient changer le cours de leurs vies. Leur empressement à accepter l'invitation à se lancer dans une telle expérience existentielle et à embrasser ses nouvelles conceptions, convainquirent Jung que ces dernières n'étaient pas purement idiosyncrasiques, mais qu'elles étaient reproductibles et possédaient une portée générale.

\footnotetext{
${ }^{71}$ Mysterium conjunctionis, GW, 14, 2, §178. Mysterium Conjunctionis, Paris, Albin Michel, 1980.

72 T. Keller, Recollections of encounter with Dr. Jung, op. cit., pp. B17-8.

${ }^{73}$ C. G. Jung, 'Ziele der Psychotherapie', GW 16, § 99. « Moyens et buts de la psychothérapie », La guérison psychologique, Genève, Georg, 1970.
} 
Cet élargissement de la fonction de la psychothérapie au-delà de la cure des pathologies eût pour conséquence que ce qui était auparavant défini comme « une méthode médicale de traitement », devint « une méthode d'auto- éducation » qui ne se limitait plus au cabinet de consulta$\operatorname{tion}^{74}$. Ceci la rapprochait des traditions ésotériques orientales d'une part, et des pratiques spirituelles européennes de l'autre. D'où l'engagement poussé de Jung, à partir des années trente, dans l'étude comparée de ces pratiques ${ }^{75}$. Il soutenait que ses fantasmes et ceux de ses patients étaient issus de l'imagination mythopoétique qui faisait défaut dans les temps présents, dominés par la rationalité. S'y reconnecter pourrait constituer les fondements d'un renouveau culturel. La tâche des modernes était d'établir le dialogue avec les contenus de l'inconscient collectif et de les intégrer à la conscience. Ceci allait jouer un rôle important dans le « renouveau mythique » populaire. Il affirma que le renouveau culturel ne pourrait avoir lieu qu'à travers l'auto- régénération de l'individu, en d'autres termes, à travers le processus d'individuation. Il proposait ce faisant, une nouvelle « image de l'homme ». Telle qu'il l'envisageait, la tâche à laquelle étaient confrontés ses patients était de recouvrer le sens de leur vie, tâche rendue de plus en plus pressante du fait de la sécularisation et de la rationalisation de la culture contemporaine. En conséquence de quoi, les individus qui parvenaient à redonner un sens à leurs vies ne faisaient pas que se guérir eux-mêmes, mais également la culture. Ainsi, le but de la cure thérapeutique n'était point d'aider le patient à s'adapter aux normes sociales existantes mais à favoriser un processus d'auto-réalisation qui, à terme, contribuerait à créer de nouvelles formes sociales. Le patient thérapeutique était devenu le médecin de la société.

\section{Traduit de l'anglais par Laurence Lacour}

\footnotetext{
${ }^{74}$ C. G. Jung, 'Die Probleme der modernen Psychotherapie,' 1929, GW 16, 174. « Les problèmes de la psychothérapie moderne », La Guérison psychologique, op. cit.

${ }^{75}$ Voir C. G. Jung, The Psychology of Kundalini Yoga: Notes of the Seminar given in 1932, édité par Sonu Shamdasani, Bollingen Series, Princeton, 1996, ainsi que la contribution de Martin Liebscher's à ce volume.
} 